\title{
Estado del conocimiento en Weinmannia tomentosa L.f. (encenillo) y algunas propuestas de estudio sobre su regeneración Current knowledge of Weinmannia tomentosa L.f. (encenillo) and some study proposals for its regeneration
}

\author{
Carmen Rosa Montes Pulido \\ carmen.montes@unad.edu.co \\ Universidad Nacional Abierta y a Distancia (UNAD) \\ Escuela de Ciencias Agrícolas Pecuarias y del Medio Ambiente (ECAPMA) \\ Sede Nacional José Celestino Mutis, Bogotá, Colombia
}

Resumen.-La reforestación de especies nativas es una prioridad de nuestros ecosistemas intervenidos o severamente afectados. $W$. tomentosa es una planta dominante en el clímax regional y muy frecuente en los bosques montañosos del norte de la cordillera de los Andes. En Colombia se encuentra principalmente en la cordillera Oriental. Es considerado como uno de los principales inductores preclimácicos de las laderas y de allí su comportamiento pionero como competidor; razón por la cual se requiere de estudios ecológicos, conocer su propagación, fisiología, entre otras. El establecimiento de $W$. tomentosa es propicio para el desarrollo de otras especies de páramo y de lugares de ladera.

Palabras clave: Propagación vegetal, Reforestación, Características ecológicas, Distribución de la especie

Abstract.- Reforestation of native species is a priority of our ecosystems intervened or severely affected. W. tomentosa is a dominant plant in the regional climax and very common in the mountainous forests of the northern Andes. In Colombia it is mainly found in the eastern cordillera. It is considered one of the main pre-climaxed slopes and hence its pioneering behavior as a competitor, that's why ecological studies are needed, to know their propagation, physiology, among others. The establishment of W. tomentosa is conducive to the development of other species of moorland and hillside locations.

Keywords: Plant propagation, Reforestation, Ecological characteristics, Specie distribution

\section{Introducción}

La especie Weinmannia tomentosa L.f. (Van Geel \& Van der Hammen 1973, citados por Cortés-S. et al. 1999), alcanzó su óptimo hace 7.000 años, una vez que la temperatura y la precipitación adquirieron los valores actuales, y reemplazó la especie Dodonaea vis$\cos a$ (L.) Jacq., extendida 12.500 años antes del presente. Sin embargo $W$. tomentosa ha sido considerablemente disminuida, debido al uso intensivo de su corteza como tanino, la copa y tronco para leña y elaboración de car- bón vegetal y empleada su madera en construcción.

La documentación sobre la regeneración del encenillo ( $W$. tomentosa) es muy escasa y se requiere información acertada para lograr mayores éxitos en la repoblación de la especie. Autores como Jaimes \& Rivera (1990) y Ramos (2001) han aportado datos sobre características de las semillas, los sitios y tendencias de su regeneración natural en bosques altoandinos aledaños al altiplano Cundiboyacense. Se han publicado documentos técnicos sobre su pro- 
pagación, fundamentados en datos empíricos obtenidos en vivero. Algunos trabajos adelantados en universidades de Colombia han avanzado sobre factores que condicionan el establecimiento de la plántula en bosques naturales; sin embargo, aún se tienen interrogantes sobre condiciones ideales para su regeneración que requieren de experimentación para ser respondidos. El objetivo de la presente revisión fue identificar y analizar estudios relacionados con los factores que condicionan el establecimiento de plántulas de encenillo.

\section{Información sobre la familia y el género}

Para Bradford \& Berry (1998) y Pennington et al. (2004), la familia Cunoniaceae presenta cerca de 25 géneros y 350 especies. La mayoría de sus géneros están presentes en el hemisferio sur, especialmente en Australia, Nueva Guinea y Nueva Caledonia. Además en Sudáfrica (Cunonia y Platyophylus), Madagascar (Weinmannia) y en América desde México hasta Chile (Weinmania, Eucryphia, Caldcluvia y Lamanonia). FernándezGaliano \& Domínguez (1985), definen como una familia de árboles y arbustos, raramente trepadoras, hojas coriáceas, opuestas (decusadas) o algunas veces verticiladas, raramente alternas, imparipinnadas, trifolioladas o pinnadas; las estípulas frecuentemente conspicuas y usualmente interpeciolares. Flores pequeñas, regulares, bisexuales o algunas veces unisexuales dioicas, solitarias o en racimos laxos o glomérulos compactos; 3-6 sépalos, libres o soldados en la base; 4-5 pétalos, libres o soldados en la base; casi siempre con numerosos estambres, pero algunas veces con 4-5 alternando con los pétalos y en otros 8-10 normalmente insertos por sus filamentos libres en una especie de disco nectarífero que rodea al ovario. El ovario es súpero, compuesto de 2-5 carpelos libres o soldados y generalmente entre 2 (algunas veces 5) cavidades con numerosos óvulos sentados en 2 filas sobre placentas encorvadas axilares o apicales. Fruto en cápsula o nuez, y semilla con embrión pequeño rodeado de abundante endospermo.
De acuerdo con Fernández-Galiano \& Domínguez (1985), el género más importante es Weinmannia que comprende 160 especies distribuidas por Madagascar, Malasia, Nueva Zelanda, Chile, México y Antillas.

El género Weinmannia, propio de bosques de alta montaña, con cerca de 77 especies en el neotrópico (Pennington et al. 2004) y aproximadamente 190 especies en el mundo (Schulze-Menz 1964, Dickison 1977). Se caracteriza por árboles y arbustos con ramitas jóvenes planas; estípulas interpeciolares, redondeadas, caducas, que tienen una cicatriz prominente; hojas opuestas, por lo general imparipinnadas opuestas, con un raquis alado, foliolos aserrados, de 1 a 3; pinnados veteados; inflorescencias axilares; por lo general en espiga o racimo; flores pequeñas, sépalos 4-5, pétalos de color blanco o rosado, de 4-5; estambres 8-10, proyectado hacia afuera; el nectario circunda el disco del ovario, ovario 2 (-3) locular, óvulos numerosos, estilos de 2 a 3 libres, estigma simple, fruto de una cápsula pequeña, septicida, de paredes delgadas, $2(-3)$, con válvula, con el estilo persistente, semillas pequeñas y peludas (Pennington et al. 2004).

\section{Las principales especies del género a nivel mundial, en Sudamérica y en Colombia}

De acuerdo con Dickison (1977), Weinmannia es el género con mayor número de especies de la familia Cunoniaceae. Su centro de distribución está en los Andes sudamericanos, pero el género alcanza a llegar hasta México (América central), Malasia, Melanesia y Polynesia, como también en el clima templado de Nueva Zelanda y Chile. El género está ausente en India, Australia y del continente africano, sin embargo, está presente en Madagascar. Aunque estas plantas son frecuentes en elevaciones medias y altas, aunque aparecen desde cerca al nivel del mar hasta los 4.000 metros de altitud (Tabla 1). 
Tabla 1. Principales especies del género Weinmannia a nivel mundial.

\begin{tabular}{|c|c|c|c|}
\hline País & Especie & Localización & Fuente \\
\hline México & W. pinnata & $\begin{array}{l}\text { 1.700- } 2.000 \text { msnm El Rincón, } \\
\text { Sierra Norte del estado de Oaxaca } \\
\text { (sureste de México) }\end{array}$ & $\begin{array}{l}\text { Bautista del Castillo \& Gutiérrez } \\
2003\end{array}$ \\
\hline Guatemala & $\begin{array}{l}\text { W. pinnata, } \\
\text { W. tuerckheimii }\end{array}$ & $\begin{array}{l}2.000-3.000 \mathrm{msnm} \\
\text { Sierra de los Cuchumatanes } \\
\text { (noroeste de Guatemala) }\end{array}$ & Islebe et al. 1994 \\
\hline Costa Rica & $\begin{array}{l}\text { W. pinnata, } \\
W . \text { trianae }\end{array}$ & $2.300-3.200 \mathrm{msnm}$ & Kappelle et al. 2000 \\
\hline Colombia & W. tomentosa & $\begin{array}{l}\text { 2.800-3.436 msnm Páramo de } \\
\text { Carmen de Carupa y Tablazo } \\
\text { (Cundinamarca); Páramo } \\
\text { Telecom (Santander); Páramo de } \\
\text { Sumapaz (Guasca) }\end{array}$ & $\begin{array}{l}\text { Arellano-P. \& Rangel-Ch. } 2008 \\
\text { Cuatrecasas (1934), Cortés- S. et } \\
\text { al. (1999) }\end{array}$ \\
\hline Colombia & W. brachystachya & $\begin{array}{l}\text { 2.800-3.436 msnm Volcan Puracé } \\
\text { (Cauca) }\end{array}$ & Arellano-P. \& Rangel-Ch. 2008 \\
\hline Colombia & W. rollotii & $\begin{array}{l}\text { 2.800-3436 msnm Páramo } \\
\text { Sumapaz (Guasca) }\end{array}$ & Arellano-P. \& Rangel-Ch. 2008 \\
\hline Colombia & W. cogolloi & $\begin{array}{l}\text { Parque Nacional Natural las } \\
\text { Orquídeas (Antioquia), sector } \\
\text { cruces, margen derecha río Calles }\end{array}$ & Morales 2005 \\
\hline Colombia & W. pinnata & Alta montaña Serranía de Perijá & Arellano-P. \& Rangel-Ch. 2008 \\
\hline Colombia & W. microphylla & $\begin{array}{l}\text { Páramo de la Rusia, Belén y } \\
\text { Guantiva (Boyacá) }\end{array}$ & Arellano-P. \& Rangel-Ch. 2008 \\
\hline Venezuela & $\begin{array}{l}\text { W. balbisiana; } \\
\text { W. brachystachya; } \\
\text { W. corocoroensis; } \\
\text { W. elliptica; } \\
\text { W. fagaroides; } \\
\text { W. glabra; } \\
\text { W. guyanensis; } \\
\text { W. ilutepuiensis; } \\
\text { W. laxiramea; } \\
\text { W. pubescens; } \\
\text { W. sorbifolia; } \\
\text { W. velutina; }\end{array}$ & $\begin{array}{l}1.200-1.900 ; \\
2.500-2.800 ; \\
2.200 \\
1.800-2.500 ; \\
1.200-1.800 ; \\
1.000-2.600 ; \\
2.200-2.800 ; \\
2.200-2.700 ; \\
2.100-2.900 ; \\
500-2.000 ; \\
1.000-1.800 \\
1.100-2.700\end{array}$ & Steyermark et al. 1998 \\
\hline Venezuela & W. tomentosa & Estados de Mérida y Táchira & Bernardi 1961 \\
\hline Venezuela & W. tomentosa & $\begin{array}{l}\text { Estados de Mérida, Táchira y Zulia } \\
2.000 \mathrm{msnm}\end{array}$ & Hokche et al. 2008 \\
\hline Ecuador & W. auriformis & $\begin{array}{l}\text { Cordillera del Cóndor y río } \\
\text { Coangos }\end{array}$ & Rogers 2002 \\
\hline Peru & $\begin{array}{l}\text { W. crassifolia, } \\
W . \text { pinnata }\end{array}$ & $2000-4.000 \mathrm{msnm}$ & Pennington et al. 2004 \\
\hline Bolivia & W. boliviana & $2.100-2.600 \mathrm{msnm}$ & Bach et al. 2003 \\
\hline Bolivia & $\begin{array}{l}\text { W. crassiflora, } \\
\text { W. microphylla }\end{array}$ & $\begin{array}{l}2.000-3.000 \text { msnm Bosque } \\
\text { Nublado }\end{array}$ & Paniagua-Zambrana et al. 2003 \\
\hline
\end{tabular}




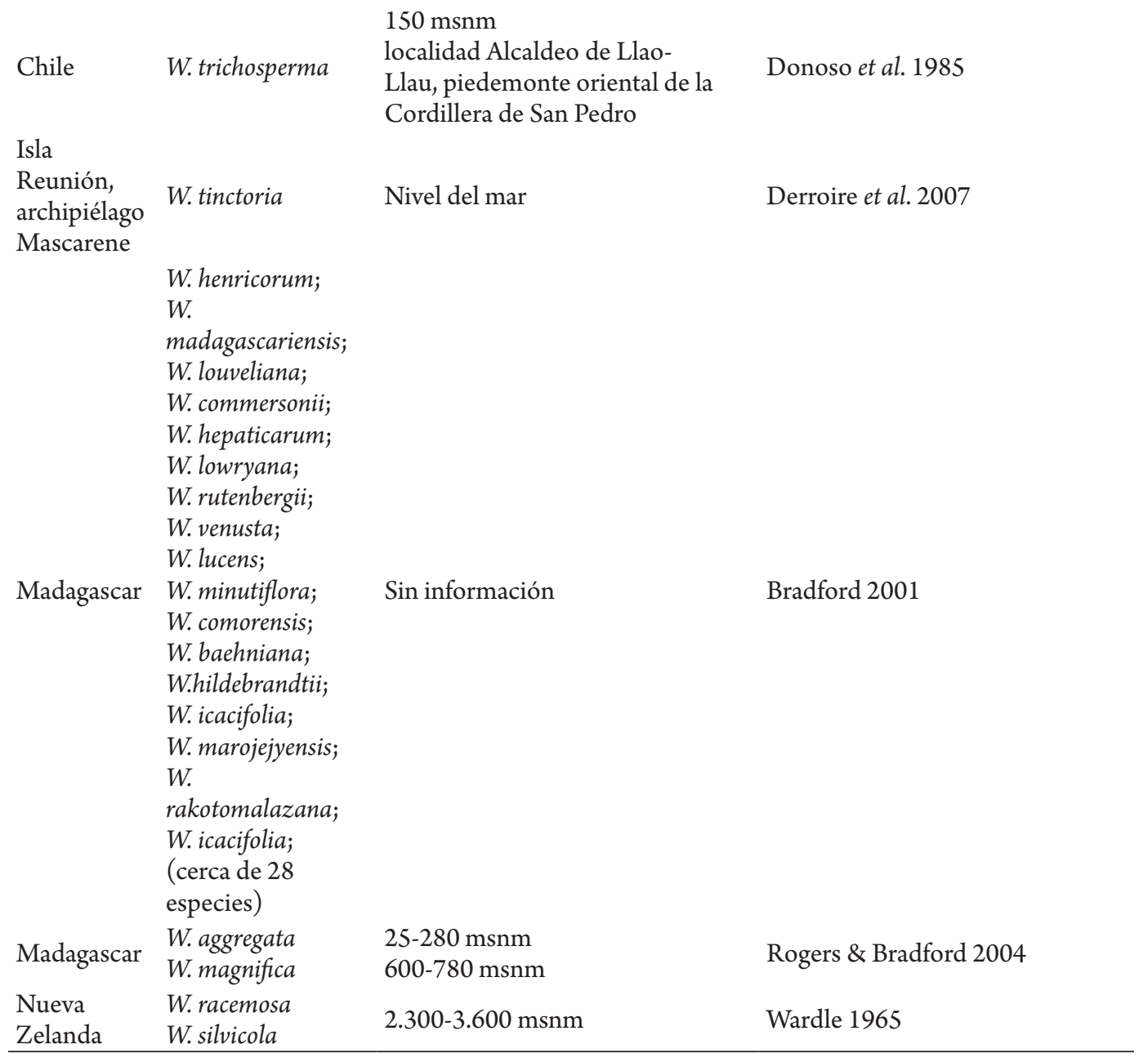

\section{Características de W.tomentosa}

Morfológicas: árbol que puede alcanzar 25 $\mathrm{m}$ de altura y $70 \mathrm{~cm}$ de diámetro en su tronco; corteza de color gris; copa en forma de parasol, semidensa y de color verde oscuro a excepción del período donde aparecen frutos que se torna café rojiza. Sus ramas crecen de manera oblicua, son de color pardo y delgadas, poseen vellos (CAR 2004).

Las hojas miden $3,8 \mathrm{~cm}$ de largo, son compuestas-opuestas; están distribuidas en cuatro filas (tetrásicas), su eje central (raquis) es alado, poseen entre cinco y ocho pares de foliolos y terminan en uno (imparipinadas), miden $1 \mathrm{~cm}$ de largo, son asimétricos, de color verde opaco por su frente. Su borde es curvado hacia su revés (revoluto), poseen vellosidades suaves (pubescentes), terminan en una punta roma y su base es oblicua; presentan estípulas connadas de color verde rojizo que albergan, antes de abrirse, insectos que depositan allí sus huevos (CAR 2004). Las inflorescencias son espigas pequeñas $(4-6 \mathrm{~cm})$ blanco-crema, al pasar a fruto se tornan rojizas y al madurar se tornan color caramelo con apariencia seca (DAMA 2000).

Los frutos miden $9 \mathrm{~mm}$ de largo y $2 \mathrm{~mm}$ de ancho; las cápsulas dehiscentes se abren en 2 
válvas, forma elíptica, color café oscuro y cada uno tiene entre 3 a 6 semillas. El color de las semillas café, miden $1 \mathrm{~mm}$ de largo y están cubiertas por vellosidades que les sirven para dispersarse por acción del viento (DAMA 2000, Rodríguez-Santamaría 2006).

\section{Características ecológicas y distribución de la especie}

El árbol crece en laderas de suelos arenosos, orgánicos, profundos y bien drenados, ocasionalmente rocosos. Aunque soporta atmósferas subhúmedas, alcanza su mayor desarrollo en los núcleos de condensación de nubes. (DAMA 2000).

La especie es dominante del conclímax regional, lo que significa, que la mayor parte de los bosques de la región estuvieron dominados por $W$. tomentosa. Se considera uno de los principales inductores preclimácicos de las laderas y requiere facilitación de precursores como tunos ( $\mathrm{Mi}$ conia spp.), romero (Pentacalia sp.), uva ( $\mathrm{Ma}$ cleania rupestris), cucharo (Myrsine guianensis) y gaques (Clusia multiflora) (DAMA 2000).

La distribución global de la especie corresponde a los bosques montañosos del norte de la Cordillera de los Andes. Los estudios revisados la reportan en la cordillera Oriental de Colombia y en los estados de Mérida, Táchira y Zulia de Venezuela (Tabla 1). Según Van der Hammen et al. \& González (1960 y 1963) y Cortés-S. et al. (1999), $W$. tomentosa y otras especies arbóreas asociadas corresponden a la vegetación original climácica más frecuente en los declives de las montañas que forman el borde oriental de la Sabana de Bogotá, en altitudes superiores a $2.800 \mathrm{msnm}$. Se encuentra mezclado con frailejonales (Espeletia sp.) y pajonales (Calamagrostis effusa) y chuscales (Chusquea tesellata) en el páramo de Carupa (Cundinamarca), en áreas de uso agropecuario entremezclado con bosques intervenidos del páramo de Tablazo (Cundinamarca) y en pajonales (Calamagrostis effusa) del páramo de Sumapaz (Cundinamarca) (Arellano \& Rangel 2008).

\section{Floración y fructificación}

Los meses que $W$. tomentosa produce flores son mayo, agosto y septiembre a octubre (CAR 2004). La fructificación se presenta en junio, septiembre y octubre a noviembre. En las zonas del Neusa y Monserrate, Ramos (2001) reporta los máximos de dispersión de semillas durante los meses de febrero, marzo y octubre a noviembre, respectivamente. La producción de semilla encontrada fue 359 semillas/sema$\mathrm{na} / \mathrm{m}^{2}$ en Neusa y 50 semillas $/$ semana $/ \mathrm{m}^{2}$ para Monserrate.

\section{Uso}

Para su uso industrial, se obtiene tanino de color rojizo para teñir pieles de animal. Igualmente se obtiene un colorante negro para entintar lana. El árbol es útil como barrera corta vientos. El extracto de sus hojas y corteza se utiliza para tratamiento de fiebres en ganado (CAR 2004).

\section{Factores que condicionan el estable- cimiento de la plántula}

Los trabajos de Jaimes \& Rivera (1990) sobre el estudio de banco de semillas y tendencias de la regeneración natural de un bosque altoandino en la región de Monserrate, identifican mediante germinación en invernadero semillas viables en muestras de suelo tomadas a diferentes profundidades. Germinaron 87 semillas viables en la hojarasca, 248 en el horizonte orgánico superficial y 29 en el horizonte orgánico medio. También encontró en parcelas de $10 \mathrm{~m}$ x 10 m 39 adultos entre 12 y 15 m de altura y solo 4 plántulas de $W$. tomentosa.

Se encontró también que $W$. tomentosa es la especie más abundante en el banco de semillas analizado (450 semillas $/ \mathrm{m}^{2}$ ); su germinación en el transcurso de 45 días fue del $80 \%$. Sin embargo, es la especie menos frecuente como plántulas en el bosque. Surge aquí la pregunta de ¿cuál es su estrategia de regeneración? a pesar que las características morfológicas de la semilla presenta tegumentos duros, reducido tamaño $(1 \mathrm{~mm})$, forma globosa con pelo y fru- 
to capsular dehiscente que facilitan su enterramiento y suelos con horizonte orgánico entre 30 y $40 \mathrm{~cm}$, y pH entre 3,3 y 4,7. Montenegro et al. (2008), estudiaron 20 atributos vitales de nueve especies leñosas en el borde del bosque altoandino en la Reserva Forestal de Cogua (Colombia) entre los 2.970 y $3.650 \mathrm{msnm}$. Los rasgos reproductivos encontrados para $W$. tomentosa reportan bajo número de semillas por fruto, semillas pequeñas, testa cubierta de pelos, ligera, dispersión anemócora y exitosa en bosque paramizado.

Otro trabajo que determinó la cantidad y calidad de la semilla de $W$. tomentosa, Ramos (2001), en dos bosques altoandinos -embalse del Neusa, sector Laureles, altitud entre 3.000$3.100 \mathrm{msnm}$ y Monserrate (cerca al páramo Granizo, La Calera) entre 3.100-3.200 msnmcon el biotipo Drimys-Weinmannia que presentaba musgos y líquenes en el estrato rasante y fuste arbóreo; epifitismo bajo a intermedio, diámetros de vegetación menores a $40 \mathrm{~cm}$ de diámetro a la altura del pecho; los árboles dominantes no superaban los $15 \mathrm{~m}$ de altura en suelos poco evolucionados, en su mayoría inceptisoles con alto contenido de materia orgánica. La precipitación en Neusa corresponde a $866,4 \mathrm{~mm}$ y Monserrate $1.079,3 \mathrm{~mm}$ anuales. La determinación de la lluvia de semillas en el sitio de Neusa, se encontró durante los meses de febrero y marzo con los mayores picos de dispersión de semillas, mientras que en Monserrate entre octubre y noviembre. Se encontraron 359 semillas/semana/ $\mathrm{m}^{2}$ en Neusa y 50 semillas/semana $/ \mathrm{m}^{2}$ en Monserrate. Las diferencias son atribuidas a factores como la pendiente, estructura de vegetación y cobertura. La dispersión nula coincide para todas las parcelas en el mes de junio. La alta dispersión de las semillas no coincide con su viabilidad, inferior al $13 \%$. La presencia de semillas vacías correspondió al $33 \%$ en predispersadas, $69 \%$ en colectores y $87 \%$ en banco de suelo. En el caso de semillas acumuladas en el suelo, entre el 85 y 94\% presentaron necrosamiento. También encontró que las semillas son poco longevas y no dormantes, por lo cual no se forma un banco de semillas propiamente dicho porque las semillas difícilmente pueden persistir meses ya formadas; la densidad de semillas es un cuantificador efímero de la potencialidad de regeneración de la especie, sin embargo, el autor afirma que si las semillas son sometidas a condiciones favorables de luz, temperatura, nutrientes y humedad, distintas a las que se dan in situ, las semillas mantienen su viabilidad un tiempo máximo de 45 dias (Jaimes \& Rivera 1990).

Además, Ramos (2001) menciona que la hojarasca presente en el suelo es un obstáculo que impide el crecimiento radicular como la emergencia de primordios foliares, debido a que funciona como un filtro de luz. Estima que las semillas permanecen viables en el suelo hasta un mes de llegada cuando comienza el necrosamiento de los tejidos debido a que son rápidamente sepultadas por el material vegetal que constantemente llega al suelo. En este sentido, revela que en $W$. tomentosa, un año después de la dispersión, aproximadamente, sólo una milésima parte de las semillas producidas, habrá llegado exitosamente al estado de semilla.

Esto sustenta las observaciones de Zuluaga citado por Ramos (2001) sobre formar grupos clonales de $W$. tomentosa a partir de un solo individuo establecido en el borde boscoso pero la habitabilidad del sitio, Ramos (2001) plantea que $W$. tomentosa tiende a ser desplazada del bosque, por no existir un banco de plántulas que reemplace a la cohorte de adultos que actualmente conforman la cobertura. A pesar que la semilla tiene la única ventaja de ser numerosa y liviana y tendría la opción de colonizar sitios de semiluz.

Por otro lado el ambiente creado por $W$. tomentosa favorece la llegada y establecimiento de otras especies de características umbrófilas y semumbrófilas del bosque andino como Drimys granadensis y Clusia multiflora. Por lo cual éstas pueden reemplazar los espacios que deja $W$. tomentosa con la mortalidad de sus adul- 
tos. El patrón sucesional sugerido por Ramos (2001) nuestra un desplazamiento de $W$. tomentosa de adentro hacia afuera del bosque. No obstante, el autor reconoce que esta especie no desaparece del ecosistema. Se comporta como árbol pionero de ciclo largo, o como una especie secundaria tardía porque invierte más energía en colonizar que en competir.

En la reproducción asexual de $W$. tomentosa se encontraron brotes axilares, en observaciones realizadas en abril de 2010, en el sitio denominado "Parque Entre Nubes" al sur oriente de Bogotá a $3.000 \mathrm{msnm}$; se desarrollaron entre dos y tres rebrotes, a manera de tallos (inicialmente rastreros y que luego se levantan en busca de luz), que sobresalen de la base del tronco, las cuales no se observan fácilmente porque se encuentran cubiertas por hojarasca, musgo y suelo orgánico. Dichos rebrotes son de diámetros variados y se encuentran a diferentes distancias, entre 0,5 a 1,0 m del tronco principal. En varios casos el tronco principal está muerto.

\section{Conclusiones}

Es conveniente recoger frutos y semillas directamente del árbol o con recipientes que eviten su caída al suelo para evitar el necrosamiento. Dado que la viabilidad de la semilla es baja y es poco longeva, se debe sembrar inmediatamente se recoge, aunque Rivera (1991) menciona un tiempo máximo de 45 días durante el que no se pierde viabilidad. Al respecto, se deben hacer ensayos de germinación que incluyan la variable tiempo durante el cual la semilla es viable.

Referente a sustratos, se parte del principio que el suelo debe ser preferiblemente orgánico, dadas las condiciones naturales descritas referentes a las características del sitio natural donde crece el $W$. tomentosa. Sin embargo, sería necesario realizar ensayos de germinación con diferentes sustratos. Por ejemplo, realizar mezclas de suelo orgánico y arena en diferentes proporciones.
Acerca del manejo de la luz se concluye que la especie prefiere para su germinación condiciones de semiluz. Atendiendo a este requerimiento, el ensayo de germinación debe determinar cuáles son esas condiciones de semiluz. Con uso de malla polisombra, dando condiciones al germinador de totalmente cubierto, 3/4, 2/4 y $1 / 4$, puede llegarse a una conclusión.

Sobre la afirmación personal proveniente de Silvio Zuluaga y referida por Ramos (2002), respecto a que $W$. tomentosa L.f. desarrolla clones, Carmen R. Montes Pulido, encuentra en observaciones realizadas en el mes de abril de 2010, en el sitio denominado "Parque Entre Nubes” al sur oriente de Bogotá sobre los 3.000 msnm, que los adultos desarrollan entre dos y tres rebrotes, a manera de tallos (inicialmente rastreros y que luego se levantan en busca de luz), que salen de la base del tronco, las cuales no se observan fácilmente porque se encuentran cubiertas por hojarasca, musgo, suelo orgánico. Dichos rebrotes son de diámetros variados y se encuentran a diferentes distancias, entre 0.50 a $1.0 \mathrm{~m}$ de distancia, respecto al tronco principal, dando la impresión de ser individuos independientes. En varios casos el tronco principal está muerto y son los rebrotes los que dan continuidad a la especie.

No se encontró publicación específica sobre propagación de $W$. tomentosa L.f. en condiciones de vivero, sin embargo el estudio de restauración adelantado por el DAMA y ASOPORQUERA reporta plantación de la especie procedente de vivero. Dicha información la poseen los trabajadores de un vivero, quienes empíricamente han logrado la germinación de ésta y otras especies nativas. Por lo anterior, sería necesario identificar el protocolo seguido en viveros de Bogotá para propagación de la especie, a fin de describir el manejo de variables de germinación como sustrato, temperatura, luz, humedad, formas de recolección de fruto, tratamiento de la semilla, tiempo de germinación desde su siembra, número de individuos germinados respecto a semillas sembradas, 
tiempos máximo y mínimo de siembra después de recolección de fruto.

Posteriormente, sería igualmente interesante realizar un ensayo de germinación "in situ" para determinar diferencias.

Finalmente, aunque $W$. tomentosa L.f. se propaga de manera empírica en vivero, ésta no es satisfactoria debido a que el número de individuos disponibles en esos espacios es mínimo e insuficiente para grandes programas de restauración ecológica de páramos. Igualmente se reconoce que una vez plantado, su crecimiento es muy lento. $\mathrm{Al}$ respecto, se desconocen los factores que pueden favorecer un rápido crecimiento.

\section{Literatura citada}

Arellano, P., H. \& J.O. Rangel-Ch. 2008. Patrones en la distribución de la vegetación en áreas de páramo de Colombia: Heterogeneidad y dependencia espacial. Caldasia 30: 355-411.

Bach, K., M. Schawe, B. Stephan, G. Gerold, S.R. Gradstein \& M. Morales R. 2003. Vegetación, suelos y clima en los diferentes pisos altitudinales de un bosque montano de Yungas, Bolivia: Primeros resultados. Ecología en Bolivia 38: 3-14.

Bautista del Castillo, R.F. \& C. Gutiérrez. 2003. Patrones de desarrollo del suelo asociados con sucesión secundaria en un área originalmente ocupada por bosque mesófilo de montaña. Revista científica y técnica de Ecología y medio ambiente 12: 1-8.

Bernardi, L. 1961. Revisio generis Weinmanniae. Pars I: Sectio Weinmanniae. Candollea 17: 123189

Bradford, C.J. \& P.E. Berry. 1998. Cunnoniaceae. En: Steyermark, J.A., P.E. Berry, K. Yatskievych \& B.K. Holst (Eds.). The flora of the Venezuelan Guyana. Vol. 4 Caesalpinaceae-Ericacea. Missouri Botanical Garden Press, Sant Louis. pp 462-469.
Bradford, J.C. 2001. The application of a cladistic analysis to the classification and identification of Weinmannia (Cunoniaceae) in Madagascar and the Comoro Islands. Adansonia, (Sér. 3) 23: 237-246.

Corporación Autónoma Regional de Cundinamarca CAR. 2004. Vegetación el territorio CAR: 450 especies de sus llanuras y montañas. Bogotá.

Cortés-S., S.P., T. Van der Hammen, \& J.O. RangelCh. 1999. Comunidades vegetales y patrones de degradación y sucesión en la vegetación de los cerros occidentales de Chía- Cundinamarca-Colombia. Revista de la Academia Colombiana de Ciencias Exactas, Físicas y Naturales 23: 529-554.

Cuatrecasas, J. 1934. Observaciones geobotánicas en Colombia. Serie Botánica No. 27. Museo Nacional de Ciencias Naturales. Madrid.

Daniel, P.W., U.E. Helms \& F.S. Baker. 1982. Principios de silvicultura. Mc Graw Hill. México.

Departamento Técnico Administrativo del Medio Ambiente DAMA. 2000. Protocolo distrital de restauración ecológica. Guía para la restauración de ecosistemas nativos en las áreas rurales de Santa Fe de Bogotá. Bogotá.

Derroire, G., L. Schmitt, J.N. Rivière, J.M. Sarrailh \& J. Tassin. 2007. The essential role of treefern trunks in the regeneration of Weinmannia tinctoria in rain forest on Réunion, Mascarene Archipielago. Journal of Tropical ecology 23: 487-492.

Dickison, W.C. 1977. Wood anatomy of Weinmannia (Cunoniaceae). Bulletin of the Torrey Botanical Club 104: 12-23.

Donoso, C., B. Escobar \& J. Urrutia. 1985. Estructura y estrategias regenerativas de un bosque virgen de Ulmo (Eucryphia cordifolia Cav.)-Tepa (Laurelia philippiana Phil) looser en Chiloè, Chile. Revista chilena de Historia natural 58: 171-186. 
Fernandez-Galiano, E. \& E. Dominguez V. 1985. Plantas con flores. Reverté, Barcelona, España. pp. 113-114.

Gomez-Restrepo, M.L. 2010. Fenología reproductiva de especies forestales nativas presentes en la jurisdicción de CORANTIOQUIA, un paso hacia su conservación. Vol. I. Corantioquia, Medellín.

Hokche, O., P.E. Berry \& O. Huber. 2008. Nuevo catalogo de la flora vascular de Venezuela. Fundación Instituto Botánico de Venezuela. Caracas.

Islebe, G.A., A.M. Cleef \& A. Velásquez. 1994. Especies leñosas de la sierra de los Cuchumatanes y de la cadena volcánica, Guatemala. Acta Botánica Mexicana 29: 83-92.

Jaimes S., V \& Rivera, O. 1990. Banco de semillas y tendencias enla regeneración natural de un bosque altoandino en la región de Monserrate (Cundinamarca, Colombia) [Tesis de grado]. Bogotá: Facultad de Ciencias, Universidad Nacional de Colombia.

Kappelle, M., L. Van Omme \& M. Juarez. 2000 Lista de la flora vascular de la cuenca superior del río Savegre, San Gerardo de Dota, Costa Rica. Acta Botánica Mexicana 51: 1-38.

Lanier, L. 1986. Précis de sylviculture. Engref, Nancy, Francia.

Montenegro, A.L. \& Vargas, O. 2008. Atributos vitales de especies leñosas en bordes de bosque altoandino de la Reserva Forestal de Cogua (Colombia). Revista de Biología Tropical 56: 705-720.

Paniagua-Zambrana, N., C. Maldonado-Goyzueta \& C. Chumacero-Moscoso. 2003. Mapa de vegetación de los alrededores de la estación Biológica de Tunquini, Bolivia. Ecología en Bolivia 38: 15-26.

Pennington, T.D. C. Reynel \& A. Daza. 2004. Ilustrated guide to the trees of Peru. David Hunt, Sherbourne, UK.
Ramos, C. 2001. Estrategias regenerativas de Clusia multiflora, Drimys granadensis y Weinmannia tomentosa L.f. en el bosque altoandino [Trabajo de grado]. Bogotá: Facultad de Ciencias, Universidad Nacional de Colombia.

Rogers, Z.S. 2002. A new Species of Weinmannia (Cunoniaceae: Cunonieae) from Southern Ecuador. Novon 12: 249-252.

Rogers, Z.S. \& J.C. Bradford. 2004. Weinmannia magnifica and $W$. aggregata (Cunoniaceae): two distinctive new species from Madagascar. Adansonia (Sér. 3) 26: 83-91.

Serrada H., R. 2003. Regeneración natural. Situaciones, concepto, factores y evaluación. Cuadernos de la Sociedad Española de Ciencias Forestales 15: 11-15.

Steyermark, J.A., P.E. Berry, K. Yatskievych \& B.K. Holst. The flora of the Venezuelan Guyana. Vol. 4 Caesalpinaceae-Ericacea. Missouri Botanical Garden Press, Sant Louis.

Van der Hammen, T. \& E. González. 1963. Historia del clima y vegetación del Pleistoceno superior y del Holoceno de la Sabana de Bogotá. Boletín Geológico 11: 189-266.

Van der Hammen, T. \& E. González. 1960. Upper Pleistocene and Holocene climate and vegetation o the Sabana de Bogotá (Colombia, South América). Leidse Geologishe Mededelingen 25: 261-315.

Wardle, P. 1965. Biological flora of New Zealand. 1. Weinmannia racemosa. New Zealand Journal of Botany abstract 4: 114-131.

Recibido: 19 de mayo de 2011 Aceptado: 14 de junio de 2011 
